exacerbations and exclusion criteria. The analysis was limited to patients aged $=45$ years to reduce contamination by COPD. Patients with non-asthma respiratory diagnoses were excluded. Results In the 3 year period about 395,000 patients received repeated medications for asthma with a monthly point prevalence of 255,000 asthma patients; equating to a prevalence of $7.0 \%$ of the 3.6 million people aged $0-45$ registered with a GP in Scotland. These patients collected 5.6 million prescriptions for inhaled/oral asthma therapies and 253,000 short courses of oral corticosteroids were dispensed to these patients in primary care. Patients were categorised on a daily basis into BTS Steps 15 according their medication use. As of June 2011: 83,663 (32.4\%) of patients were in Step 1;99,374 (38.5\%) were in Step 2; 42,555, (16.5\%) were Step 3; 27852 (10.8\%) were Step 4 , and 4,500 patients $(1.7 \%)$ were Step 5 receiving daily oral corticosteroids. Table 1 presents the exacerbation rates for these patients, $1.9 \%$ of the patients received an emergency short course of prednisolone,. Conclusion This current and whole population database indicates that in Scotland asthma prevalence is about $7 \%$ in this age group. The proportion of asthma patients treated at Steps 3-5 (25\%) is greater than the often quoted $15 \%$. Although proportionately small, a sizeable number of patients $(\sim 7000)$ with Step 1 and 2 asthma had at least one exacerbation requiring short course prednisolone and/or hospital contact over a three year period.

$\begin{aligned} & \text { Abstract P16 Table 1. Dispensed inhaled therapy and A\&E } \\
& \text { attendance data from the Scottish NHS databases }\end{aligned}$
\begin{tabular}{llllll}
\hline BTS Steps & $\mathbf{1}$ & $\mathbf{2}$ & $\mathbf{3}$ & $\mathbf{4}$ & $\mathbf{5}$ \\
\hline $\mathrm{N}$ & 83,663 & 99,374 & 42,555 & 27,852 & 4,500 \\
$\% \geq 1$ short-course prednisolone & $1.0 \%$ & $2.0 \%$ & $2.2 \%$ & $4.3 \%$ & $0.5 \%$ \\
$\% \geq 1$ A\&E attendance with asthma over 3 years & $1.4 \%$ & $2.7 \%$ & $3.3 \%$ & $4.5 \%$ & $10.9 \%$ \\
\hline
\end{tabular}

\section{P17 THE BURDEN OF ICS/LABA-TREATED ASTHMA PATIENTS IN THE UK ADULT POPULATION}

${ }^{1} \mathrm{D}$ Price, ${ }^{2} \mathrm{~N}$ Mathieson, ${ }^{2} \mathrm{~A}$ Mulgirigama, ${ }^{2} \mathrm{~A}$ Scowcroft, ${ }^{3} \mathrm{R}$ Pedersini, ${ }^{3} \mathrm{G}$ Isherwood, ${ }^{4}$ ID Pavord; ' $A$ Academic Centre of Primary Care, University of Aberdeen, Aberdeen, UK; ${ }^{2}$ Boehringer Ingelheim Ltd UK, Berkshire, UK; ${ }^{3}$ Kantar Health, Epsom, UK; ${ }^{4}$ University Hospitals of Leicester NHS Trust, Institute for Lung Health, Glenfield Hospital, Leicester, UK

\subsection{6/thoraxjn-2013-204457.167}

Objectives According to NHS QOF (Quality and Outcomes Framework) figures, 3.3 million UK citizens have asthma. Previous studies have shown an association of asthma with increased direct and indirect healthcare costs, but similar studies have not been conducted specifically for UK asthma patients. The aim of the current study is to assess the impact of poor asthma control on UK patients treated with ICS + LABA maintenance treatment.

Methods Data were from the 2010 and 2011 UK National Health and Wellness Survey (NHWS), an Internet-based questionnaire from a representative sample of UK adults stratified by age and gender. 701 respondents self-reported a diagnosis of asthma without concomitant COPD, chronic bronchitis, or emphysema and were currently being treated with ICS + LABA.

Patients Not Well Controlled (NWC) according to ACT (score $<20$ ) were compared to well-controlled (WC) patients (score $\geq 20$ ) on demographics, medications, health status, BMI, comorbidities, adherence (MMAS-4), healthcare use (number of physician visits, emergency visits and hospitalizations), work productivity and activity impairment (WPAI) and health-related quality of life (HR-QoL) (SF-12).

Results A greater proportion of the 452 NWC patients (64\% of the overall sample) go to emergency $(21 \%$ vs. $14 \%, \mathrm{p}=0.016)$ or are hospitalised $(13 \%$ vs. $8 \%, \mathrm{p}=0.022)$, in comparison with the WC; Their mental and physical HR-QoL is lower (SF12 MCS: 43 vs. $47 / 100$; PCS: 40 vs. $48 / 100$; Health utility: 0.65 vs. $0.74 / 1.00$; all p's $<0.001$ ); while their work and activity impairment are greater: presenteeism $(23 \%$ vs. $11 \%, \mathrm{p}<$ $0.001)$, overall work impairment $(29 \%$ vs. $17 \%, \mathrm{p}<0.001)$ and activity impairment $(46 \%$ vs. $24 \%, \mathrm{p}<0.001)$. In the current sample, NWC did not show significantly different levels of adherence from WC (50\% vs. $55 \%, \mathrm{p}=0.361)$.

Conclusions Over 60\% UK ICS + LABA-treated adult patients are poorly controlled. Poor control is associated with lower HRQoL, greater healthcare use and productivity impairment, but not with significantly different levels of adherence to WC patients. The recognition of patients remaining symptomatic and utilising healthcare resource whilst treated with ICS + LABA maintenance therapy is an important step to improving their management.

\section{P18 IDIOPATHIC PULMONARY FIBROSIS SURVIVAL HAS NOT IMPROVED IN THE 21ST CENTURY; ANALYSIS OF CPRD GOLD PRIMARY CARE DATA}

${ }^{1}$ Tm Maher, ${ }^{2} \mathrm{H}$ Strongman, ${ }^{2} \mathrm{R}$ Boggon, ${ }^{3} \mathrm{I}$ Kausar; ${ }^{1}$ Royal Brompton Hospital, London, United Kingdom; ${ }^{2}$ Clinical Practice Research Datalink, London, United Kingdom; ${ }^{3}$ InterMune UK and Ireland, London, United Kingdom

\subsection{6/thoraxjnl-2013-204457.168}

Introduction and Objectives Idiopathic pulmonary fibrosis (IPF) is a progressive and invariably fatal disease. Historical cohort studies have reported a median survival of $2.8-3.2$ years $^{1}$. The UK has seen a number of important developments for IPF patients in the last year which it is hoped will translate to improved outcomes for individuals with this devastating disease. These include: the licensing and approval by NICE of the first anti fibrotic therapy, pirfenidone; specialist commissioning of ILD services; and publication of NICE IPF guidelines. If the impact of these important developments is to be measured it is vital that current IPF disease burden is understood.

Methods The incidence and mortality of IPF-Clinical Syndrome, using broad and narrow disease definitions, was assessed via the Clinical Practice Research Datalink (CPRD) GOLD dataset.

Results The incidence of broadly defined IPF-CS between 2000-2012 was 8.65 (95\% CI 8.40-8.90) per 100,000 person years and significantly increased over time (incidence rate ratio adjusted by gender, region and age category $=1.02$, $\mathrm{p}$ for linear trend $<0.0001$ ). Kaplan-Meier survival estimates show no significant survival difference by year of diagnosis (log rank test for equality of survivor functions, $\mathrm{p}=0.17$ ) with a median survival of 3.0 years (95\% CI 2.8-3.1). There was no significant survival difference for broad and narrow definitions (log rank test for equality of survivor functions, $\mathrm{p}=0.06$ ) validating the definition of IPF-CS.

Conclusions In keeping with previous data the incidence of IPFCS continues to rise and the survival time from diagnosis remains unchanged. The mortality burden of IPF-CS is therefore increasing. The results of this study provide an important benchmark against which the effects of changes in the management and delivery of care for individuals with IPF can be measured. 


\section{REFERENCES}

1. Navaratnam V, Fleming KM, West J, Smith CJ, Jenkins RG, Fogarty A, Hubbard RB. The rising incidence of idiopathic pulmonary fibrosis in the U. K. Thorax. 2011;66:462-7.

\section{P19 WORRYING TREND OF LABELLING AMBIGUOUS DEATHS AS PNEUMONIA AND POTENTIAL IMPACT ON RESPIRATORY SERVICE IN A DISTRICT GENERAL HOSPITAL}

${ }^{1} E L$ Tan, ${ }^{2}$ E Davies, ${ }^{3} \mathrm{~S}$ Javed, ${ }^{2} \mathrm{R}$ Sundar, ${ }^{2}$ I Aziz; ${ }^{1}$ East Lancashire Hopitals, Blackburn, UK; ${ }^{2}$ Respiratory Medicine, Royal Albert Edward Infirmary, Wigan, UK; ${ }^{3}$ School of Medicine, University of Manchester, Manchester, UK

\subsection{6/thoraxjnl-2013-204457.169}

Background Pneumonia is a common cause of death recorded on death certificates. This data is used by Dr Foster to calculate hospital specific mortality rate (HSMR). However there is a general impression that pneumonia is recorded as the cause of death without confirmation. The British Thoracic Society (BTS) defines pneumonia as symptoms and signs consistent with an acute lower respiratory tract infection associated with new radiographic shadowing for which there is no other explanation' aspiration pneumonia 20 patients, bronchopneumonia 8 patients, community acquired pneumonia 19 patients, pneumonia 44 patients and hospital acquired pneumonia 20 patients. Out of these 111 patients, $75(67.6 \%)$ patients had radiological changes consistent with a diagnosis of pneumonia on CXR. Out of these 75 patients with radiologically confirmed pneumonia 29 $(38.7 \%)$ were given incorrect antibiotics as they were treated mainly for sepsis.

Conclusions Our findings show a very worrying trend of incorrectly recording pneumonia as cause of death in a third of patients, who were given pneumonia as cause of death. This would increase the HSMR for pneumonia as calculated by Dr Foster. In our opinion pneumonia as a cause of death is an easy option for many medical practitioners.

Recommendations We recommend an early input by respiratory physicians for all respiratory admisssions to make sure that respiratory illnesses are managed correctly.

\section{REFERENCES}

1. Thorax 2009; 64 (Supplement III) : 1-61.

\section{P20 DOES CASE ACQUISITION BIAS CONTRIBUTE TO HIGHER THAN EXPECTED MORTALITY RATES IN THE BTS NATIONAL AUDIT OF COMMUNITY ACQUIRED PNEUMONIA (CAP)?}

C Sharp, M Hameed, BD Patel; Royal Devon and Exeter NHS Foundation Trust, Exeter, United Kingdom

\subsection{6/thoraxjnl-2013-204457.170}

Background The BTS CAP audit is the largest national audit of adult CAP management. It relies on acute trusts entering data collected retrospectively from patients' notes. Cases are selected for entry if they were admitted between $1^{\text {st }}$ Dec 2012 and $31^{\text {st }}$ Jan 2013 with a diagnosis of CAP confirmed by appropriate radiological changes. Participating institutions are required to submit a minimum of 20 cases. The audit also provides data on mortality. The national data shows a high mortality rate (18.2\%) for CAP. However, the mortality data from our own institution was unexpectedly higher at $28.2 \%$.
Aim To determine if the high mortality rate from our institution is the result of selection bias.

Methods We compared the outcomes of 39 audit cases entered from our institution, a large teaching hospital serving a semi-rural population, with all other cases of CAP admitted over the same period not entered into the audit. Proportions were compared using chi-square tests and continuous variables using Kruskal-Wallis test.

Results During the two month audit period, 124 cases of CAP were identified of whom $39(31.5 \%)$ were entered into the audit. There was no significant difference in age between those entered (77.1 yrs \pm SD 11.0) and those not entered (70.1 yrs \pm 18.5 ). However the inpatient mortality rate was significantly higher in those entered into the audit than those who were not $(28.2 \%$ Vs $10.6 \%, \mathrm{p}=0.01)$.

Conclusions These results show that selection bias accounts for the apparently high mortality rates. Although the notes of all patients admitted with CAP were requested for the audit, on reviewing the methods used by the audit department, it is apparent that patients whose notes are most readily available are collected first for audit. Deceased patients' notes are more easily accessed by the audit team; since the national audit requires only a proportion of patients to be entered, this group are over-represented. If other institutions have similar practices, the national audit will over-estimate mortality from CAP. Case acquisition bias could be reduced by collecting cases prospectively, or by entering all cases of CAP over a shorter predefined time period.

\section{P21 THE RELATIONSHIP BETWEEN EMPLOYMENT STATUS, WORK PRODUCTIVITY AND QUALITY OF LIFE AMONG PATIENTS WITH COPD: CROSS-SECTIONAL ANALYSIS OF THE BIRMINGHAM COPD COHORT}

${ }^{1} \mathrm{~K}$ Kalirai, ${ }^{2} \mathrm{P}$ Adab, ${ }^{2} \mathrm{R}$ Jordan, ${ }^{2} \mathrm{D}$ Fitzmaurice, ${ }^{1} \mathrm{~J}$ Ayres; ${ }^{1}$ Institute of Occupational and Environmental Medicine, The University of Birmingham, Birmingham, England; ${ }^{2}$ Public Health, Epidemiology \& Biostatistics, The University of Birmingham, Birmingham, England

\subsection{6/thoraxjnl-2013-204457.171}

Introduction Health related quality of life (HRQoL) tends to be lower among COPD patients, as a result of associated symptoms, comorbidities and the impact of disease on daily life. However, little is known about the association between employment status and HRQoL among COPD patients.

Aims We examined the relationships between HRQoL and employment status and occupational performance among patients with COPD.

Methods 2000 patients with COPD from primary care are being recruited into the Birmingham COPD cohort study. In addition to clinical data, employment status, work performance and HRQoL were assessed. Work performance was measured using the SPS-6 presenteeism questionnaire. HRQoL was measured using the St George's respiratory questionnaire (SGRQ-C). Interim baseline data was used to assess associations between HRQoL and employment status and HRQoL and occupational performance. Multivariate analyses were used to adjust for potential confounders. Model 1 adjusted for age, sex and smoking status. Model 2 additionally adjusted for disease severity (GOLD stage), number of co-morbidities and MRC dyspnoea score.

Results Of the 1094 patients recruited, 14.6\% ( $n=160)$ were in work. Employment status: Model 1 showed that poorer quality of life was associated with lower likelihood of being in employment $(\mathrm{OR}=0.98,95 \%$ CI $0.96-0.99)$, but the effect 